Bull. Korean Math. Soc. 52 (2015), No. 1, pp. 1-11

http://dx.doi.org/10.4134/BKMS.2015.52.1.001

\title{
HAYMAN $T$ DIRECTIONS OF MEROMORPHIC FUNCTIONS IN SOME ANGULAR DOMAINS
}

\author{
NAN Wu And Zu-Xing Xuan
}

\begin{abstract}
This paper is devoted to investigate the singular directions of meromorphic functions in some angular domains. We will confirm the existence of Hayman $T$ directions in some angular domains. This is a continuous work of Yang [8] and Zheng [10].
\end{abstract}

\section{Introduction and main results}

Let $f(z)$ be a meromorphic function on the whole complex plane. We will use the standard notation of the Nevanlinna theory of meromorphic functions, such as $T(r, f), N(r, f), m(r, f), \delta(a, f)$. For the detail, see [7]. The order and lower order of it are defined as follows

$$
\lambda(f)=\limsup _{r \rightarrow \infty} \frac{\log T(r, f)}{\log r}
$$

and

$$
\mu(f)=\liminf _{r \rightarrow \infty} \frac{\log T(r, f)}{\log r} .
$$

In view of the second fundamental theorem of Nevanlinna, Zheng [11] introduced a new singular direction, which is named $T$ direction.

Definition 1.1. A direction $L: \arg z=\theta$ is called a $T$ direction of $f(z)$ if for any $\varepsilon>0$, we have

$$
\limsup _{r \rightarrow \infty} \frac{N\left(r, Z_{\varepsilon}(\theta), f=a\right)}{T(r, f)}>0
$$

for all but at most two values of $a$ in the extended complex plane $\widehat{\mathbb{C}}$. Here

$$
N(r, \Omega, f=a)=\int_{1}^{r} \frac{n(t, \Omega, f=a)}{t} d t,
$$

Received December 23, 2011; Revised May 23, 2014

2010 Mathematics Subject Classification. Primary 30D10; Secondary 30D20, 30B10, 34M05.

Key words and phrases. Hayman $T$ direction, angular domain, Pólya peaks, order. 
where $n(t, \Omega, f=a)$ is the number of the roots of $f(z)=a$ in $\Omega \cap\{1<|z|<t\}$, counted according to multiplicity. And through out this paper, we denote $Z_{\varepsilon}(\theta)=\{z: \theta-\varepsilon<\arg z<\theta+\varepsilon\}$ and $\Omega(\alpha, \beta)=\{z: \alpha<\arg z<\beta\}$.

The reason about the name is that we use the Nevanlinna's characteristic $T(r, f)$ as the comparison function. Under the growth condition

$$
\limsup _{r \rightarrow \infty} \frac{T(r, f)}{(\log r)^{2}}=+\infty
$$

Guo, Zheng and Ng [2] confirmed the existence of this singular direction and they pointed out the growth condition (1.1) is sharp. Later, Zhang [9] showed that $T$ directions are different from Borel directions whose definition can be found in [3].

In 1979, Yang [8] showed the following theorem, which provides a condition for an angular domain to contain at least one Borel direction.

Theorem A. Let $f(z)$ be a meromorphic function on the whole complex plane, with $\mu<\infty, 0<\lambda \leq \infty$. Let $\rho$ be a finite number such that $\lambda \geq \rho \geq \mu$ and $\rho>1 / 2$. If $f^{(k)}(z)(k \geq 0)$ has $p$ distinct deficient values $a_{1}, a_{2}, \ldots, a_{p}$, then in any angular domain $\Omega(\alpha, \beta)$ such that

$$
\beta-\alpha>\max \left\{\frac{\pi}{\rho}, 2 \pi-\frac{4}{\rho} \sum_{i=1}^{p} \arcsin \sqrt{\frac{\delta\left(a_{i}, f^{(k)}\right)}{2}}\right\},
$$

$f(z)$ has a Borel direction with order $\geq \rho$.

Recently, Zheng [10] discussed the problem of $T$ directions of a meromorphic function in one angular domain by proving.

Theorem B. Let $f(z)$ be a transcendental meromorphic function with finite lower order $\mu$ and non-zero order $\lambda$ and $f$ has a Nevanlinna deficient value $a \in \widehat{\mathbb{C}}$ with $\delta=\delta(a, f)>0$. For any positive and finite $\tau$ with $\mu \leq \tau \leq \lambda$, consider the angular domain $\Omega(\alpha, \beta)$ with

$$
\beta-\alpha>\max \left\{\frac{\pi}{\tau}, 2 \pi-\frac{4}{\tau} \arcsin \sqrt{\frac{\delta}{2}}\right\} .
$$

Then $f(z)$ has a $T$ direction in $\Omega=\Omega(\alpha, \beta)$.

Following Yang [8] and Zheng [10], we will continue the discussion of singular directions of $f(z)$ in some angular domains. The following three questions will be investigated in this paper.

Question 1.1. Can we extend Theorem B to some angular domains

$$
X=\bigcup_{j=1}^{q}\left\{z: \alpha_{j} \leq \arg z \leq \beta_{j}\right\}
$$

where the q pair of real numbers $\left\{\alpha_{j}, \beta_{j}\right\}$ satisfy

$$
-\pi \leq \alpha_{1}<\beta_{1} \leq \alpha_{2}<\beta_{2} \leq \cdots \leq \alpha_{q}<\beta_{q} \leq \pi ?
$$


Question 1.2. Can $f(z)$ in Theorem $B$ be replaced by any derivative $f^{(p)}(z)$ $(p \geq 0)$ ?

Question 1.3. What can we do if $f(z)$ has many deficient values $a_{1}, a_{2}, a_{3}, \ldots$, $a_{l}$ in Theorem B?

According to the Hayman inequality (see [3]) on the estimation of $T(r, f)$ in terms of only two integrated counting functions for the roots of $f(z)=a$ and $f^{(k)}(z)=b$ with $b \neq 0$, Guo, Zheng and Ng proposed in [2] a singular direction named Hayman $T$ direction as follows.

Definition 1.2. Let $f(z)$ be a transcendental meromorphic function. A direction $L: \arg z=\theta$ is called a Hayman $T$ direction of $f(z)$ if for any small $\varepsilon>0$, any positive integer $k$ and any complex numbers $a$ and $b \neq 0$, we have

$$
\limsup _{r \longrightarrow \infty} \frac{N\left(r, Z_{\varepsilon}(\theta), f=a\right)+N\left(r, Z_{\varepsilon}(\theta), f^{(k)}=b\right)}{T(r, f)}>0 .
$$

Recently, Zheng and the first author [12] confirmed the existence of Hayman $T$ direction under the condition that

$$
\limsup _{r \rightarrow+\infty} \frac{T(r, f)}{(\log r)^{3}}=+\infty
$$

In the same paper, the authors pointed out the Hayman $T$ direction is different from the $T$ direction and they gave an example to show the growth condition (1.3) is sharp. Can we discuss the problem of the existence of Hayman $T$ direction in some angular domains in the viewpoint of Questions 1.1-1.3? Though out this paper, we define

$$
\omega=\max \left\{\frac{\pi}{\beta_{1}-\alpha_{1}}, \ldots, \frac{\pi}{\beta_{q}-\alpha_{q}}\right\} .
$$

Now, we state our theorems as follows.

Theorem 1.1. Let $f(z)$ be a transcendental meromorphic function with finite lower order $\mu<\infty, 0<\lambda \leq \infty$. There is an integer $p \geq 0$, such that $f^{(p)}$ has a Nevanlinna deficient value $a \in \widehat{\mathbb{C}}$ with $\delta\left(a, f^{(p)}\right)>0$. For $q$ pairs of real numbers satisfies (1.2), $f$ has at least one Hayman $T$ direction in $X$ if

$$
\sum_{j=1}^{q}\left(\alpha_{j+1}-\beta_{j}\right)<\frac{4}{\sigma} \arcsin \sqrt{\frac{\delta\left(a, f^{(p)}\right)}{2}}
$$

where $\mu \leq \sigma \leq \lambda$, and $\omega<\sigma$.

Theorem 1.2. Let $f(z)$ be a transcendental meromorphic function with finite lower order $\mu<\infty, 0<\lambda \leq \infty$. There is an integer $p \geq 0$, such that $f^{(p)}$ has $l \geq 1$ distinct deficient values $a_{1}, a_{2}, \ldots, a_{l}$ with the corresponding deficiency 
$\delta\left(a_{1}, f^{(p)}\right), \delta\left(a_{2}, f^{(p)}\right), \ldots, \delta\left(a_{l}, f^{(p)}\right)$. For $q$ pair of real numbers $\left\{\alpha_{j}, \beta_{j}\right\}$ satisfying (1.2) and

$$
\sum_{j=1}^{q}\left(\alpha_{j+1}-\beta_{j}\right)<\sum_{j=1}^{l} \frac{4}{\sigma} \arcsin \sqrt{\frac{\delta\left(a_{j}, f^{(p)}\right)}{2}},
$$

where $\mu \leq \sigma \leq \lambda$. If $\omega<\sigma$, then $f$ has at least one Hayman $T$ direction in $X$.

We will only prove Theorem 1.2, and Theorem 1.1 is a special case of Theorem 1.2.

\section{Primary knowledge and some lemmas}

In order to prove the theorems, we give some lemmas. The following result is from [11].

Lemma 2.1. Let $f(z)$ be a transcendental meromorphic function with lower order $\mu<\infty$ and order $0<\lambda \leq \infty$. Then for any positive number $\mu \leq \sigma \leq \lambda$ and a set $E$ with finite measure, there exists a sequence $\left\{r_{n}\right\}$, such that

(1) $r_{n} \notin E, \lim _{n \rightarrow \infty} \frac{r_{n}}{n}=\infty$;

(2) $\liminf _{n \rightarrow \infty} \frac{\log T\left(r_{n}, f\right)}{\log r_{n}} \geq \sigma$

(3) $T(t, f)<(1+o(1))\left(\frac{2 t}{r_{n}}\right)^{\sigma} T\left(r_{n} / 2, f\right), t \in\left[r_{n} / n, n r_{n}\right]$;

(4) $T(t, f) / t^{\sigma-\varepsilon_{n}} \leq 2^{\sigma+1} T\left(r_{n}, f\right) / r_{n}^{\sigma-\varepsilon_{n}}, 1 \leq t \leq n r_{n}, \varepsilon_{n}=[\log n]^{-2}$.

We recall that $\left\{r_{n}\right\}$ is called the Pólya peaks of order $\sigma$ outside $E$. Given a positive function $\Lambda(r)$ satisfying $\lim _{r \rightarrow \infty} \Lambda(r)=0$. For $r>0$ and $a \in \mathbb{C}$, define

$$
D_{\Lambda}(r, a)=\left\{\theta \in[-\pi, \pi): \log ^{+} \frac{1}{\left|f\left(r e^{i \theta}\right)-a\right|}>\Lambda(r) T(r, f)\right\},
$$

and

$$
D_{\Lambda}(r, \infty)=\left\{\theta \in[-\pi, \pi): \log ^{+}\left|f\left(r e^{i \theta}\right)\right|>\Lambda(r) T(r, f)\right\} .
$$

The following result is called the generalized spread relation, and Wang in [6] proved this.

Lemma 2.2. Let $f(z)$ be transcendental and meromorphic in $\mathbb{C}$ with the finite lower order $\mu<\infty$ and the positive order $0<\lambda \leq \infty$ and has $l \geq 1$ distinct deficient values $a_{1}, a_{2}, \ldots, a_{l}$. Then for any sequence of Pólya peaks $\left\{r_{n}\right\}$ of order $\sigma>0, \mu \leq \sigma \leq \lambda$ and any positive function $\Lambda(r) \rightarrow 0$ as $r \rightarrow+\infty$, we have

$$
\liminf _{n \rightarrow \infty} \sum_{j=1}^{l} \operatorname{meas} D_{\Lambda}\left(r_{n}, a_{j}\right) \geq \min \left\{2 \pi, \frac{4}{\sigma} \sum_{j=1}^{l} \arcsin \sqrt{\frac{\delta\left(a_{j}, f^{(p)}\right)}{2}}\right\} .
$$

From [8], we know that if $a \neq b$ are two deficient values of $f$, then we have $D_{\Lambda}(r, a) \bigcap D_{\Lambda}(r, b)=\emptyset$. 
Nevanlinna theory on the angular domain plays an important role in this paper. Let us recall the following terms:

$$
\begin{aligned}
& A_{\alpha, \beta}(r, f)=\frac{\omega}{\pi} \int_{1}^{r}\left(\frac{1}{t^{\omega}}-\frac{t^{\omega}}{r^{2 \omega}}\right)\left\{\log ^{+}\left|f\left(t e^{i \alpha}\right)\right|+\log ^{+}\left|f\left(t e^{i \beta}\right)\right|\right\} \frac{d t}{t}, \\
& B_{\alpha, \beta}(r, f)=\frac{2 \omega}{\pi r^{\omega}} \int_{\alpha}^{\beta} \log ^{+}\left|f\left(r e^{i \theta}\right)\right| \sin \omega(\theta-\alpha) d \theta, \\
& C_{\alpha, \beta}(r, f)=2 \sum_{1<\left|b_{n}\right|<r}\left(\frac{1}{\left|b_{n}\right|^{\omega}}-\frac{\left|b_{n}\right|^{\omega}}{r^{2 \omega}}\right) \sin \omega\left(\theta_{n}-\alpha\right),
\end{aligned}
$$

where $\omega=\frac{\pi}{\beta-\alpha}$, and $b_{n}=\left|b_{n}\right| e^{i \theta_{n}}$ is a pole of $f(z)$ in the angular domain $\Omega(\alpha, \beta)$, appeared according to the multiplicities. The Nevanlinna's angular characteristic is defined as follows:

$$
S_{\alpha, \beta}(r, f)=A_{\alpha, \beta}(r, f)+B_{\alpha, \beta}(r, f)+C_{\alpha, \beta}(r, f) .
$$

From the definition of $B_{\alpha, \beta}(r, f)$, we have the following inequality, which will be used later.

$$
B_{\alpha, \beta}(r, f) \geq \frac{2 \omega \sin (\omega \varepsilon)}{\pi r^{\omega}} \int_{\alpha+\varepsilon}^{\beta-\varepsilon} \log ^{+}\left|f\left(r e^{i \theta}\right)\right| d \theta .
$$

The following is the Nevanlinna first and second fundamental theorem on the angular domains.

Lemma 2.3. Let $f$ be a nonconstant meromorphic function on the angular domain $\Omega(\alpha, \beta)$. Then for any complex number $a$,

$$
S_{\alpha, \beta}(r, f)=S_{\alpha, \beta}\left(r, \frac{1}{f-a}\right)+O(1), r \rightarrow \infty,
$$

and for any $q(\geq 3)$ distinct points $a_{j} \in \widehat{\mathbb{C}}(j=1,2, \ldots, q)$,

$$
(q-2) S_{\alpha, \beta}(r, f) \leq \sum_{j=1}^{q} \bar{C}_{\alpha, \beta}\left(r, \frac{1}{f-a_{j}}\right)+Q_{\alpha, \beta}(r, f),
$$

where

$$
Q_{\alpha, \beta}(r, f)=(A+B)_{\alpha, \beta}\left(r, \frac{f^{\prime}}{f}\right)+\sum_{j=1}^{q}(A+B)_{\alpha, \beta}\left(r, \frac{f^{\prime}}{f-a_{j}}\right)+O(1) .
$$

The key point is the estimation of error term $Q_{\alpha, \beta}(r, f)$, which can be obtained for our purpose of this paper as follows. And the following is true (see [1]). Write

$$
Q(r, f)=A_{\alpha, \beta}\left(r, \frac{f^{(p)}}{f}\right)+B_{\alpha, \beta}\left(r, \frac{f^{(p)}}{f}\right) .
$$

Then

(1) $Q(r, f)=O(\log r)$ as $r \rightarrow \infty$, when $\lambda(f)<\infty$. 
(2) $Q(r, f)=O(\log r+\log T(r, f))$ as $r \rightarrow \infty$ and $r \notin E$ when $\lambda(f)=\infty$, where $E$ is a set with finite linear measure.

The following result is useful for our study, the proof of which is similar to the case of the characteristic functions $T(r, f)$ and $T\left(r, f^{(k)}\right)$ on the whole complex plane. For the completeness, we give out the proof.

Lemma 2.4. Let $f(z)$ be a meromorphic function on the whole complex plane. Then for any angular domain $\Omega(\alpha, \beta)$, we have

$$
S_{\alpha, \beta}\left(r, f^{(p)}\right) \leq(p+1) S_{\alpha, \beta}(r, f)+O(\log r+\log T(r, f)),
$$

possibly outside a set of $r$ with finite measure.

Proof. In view of the definition of $S_{\alpha, \beta}(r, f)$ and Lemma 2.3, we get the following

$$
\begin{aligned}
S_{\alpha, \beta}\left(r, f^{(p)}\right) & \leq C_{\alpha, \beta}\left(r, f^{(p)}\right)+(A+B)_{\alpha, \beta}(r, f)+(A+B)_{\alpha, \beta}\left(r, \frac{f^{(p)}}{f}\right) \\
& =p \bar{C}_{\alpha, \beta}(r, f)+S_{\alpha, \beta}(r, f)+(A+B)_{\alpha, \beta}\left(r, \frac{f^{(p)}}{f}\right) \\
& \leq(p+1) S_{\alpha, \beta}(r, f)+Q(r, f) .
\end{aligned}
$$

Recall the definition of Ahlfors-Shimizu characteristic in an angle (see [5]). Let $f(z)$ be a meromorphic function on an angle $\Omega=\{z: \alpha \leq \arg z \leq \beta\}$. Set $\Omega(r)=\Omega \cap\{z: 1<|z|<r\}$. Define

$$
\mathcal{S}(r, \Omega, f)=\frac{1}{\pi} \iint_{\Omega(r)}\left(\frac{\left|f^{\prime}(z)\right|}{1+|f(z)|^{2}}\right)^{2} d \sigma
$$

and

$$
\mathcal{T}(r, \Omega, f)=\int_{1}^{r} \frac{\mathcal{S}(t, \Omega, f)}{t} d t .
$$

The following lemma is a theorem in [12], which allows one to control the term $\mathcal{T}\left(r, \Omega_{\varepsilon}\right)$ using the counting functions $N(r, \Omega, f=a)$ and $N\left(r, \Omega, f^{(k)}=b\right)$.

Lemma 2.5. Let $f(z)$ be meromorphic in an angle $\Omega=\{z: \alpha \leq \arg z \leq \beta\}$. Then for any small $\varepsilon>0$, any positive integer $k$ and any two complex numbers $a$ and $b \neq 0$, we have

$$
\mathcal{T}\left(r, \Omega_{\varepsilon}, f\right) \leq K\left\{N(2 r, \Omega, f=a)+N\left(2 r, \Omega, f^{(k)}=b\right)\right\}+O\left(\log ^{3} r\right)
$$

for a positive constant $K$ depending only on $k$, where $\Omega_{\varepsilon}=\{z: \alpha+\varepsilon<\arg z<$ $\beta-\varepsilon\}$.

In order to prove our theorem, we have to use the following lemma, which is a consequent result of Theorem 3.1.6 in [10].

Lemma 2.6. Let $f(z)$ be a transcendental meromorphic function in the whole plane, and satisfy the conditions of Theorem 1.2 or Theorem 1.1. Take a sequence of Pólya peak $\left\{r_{n}\right\}$ of $f(z)$ of order $\sigma>\omega=\frac{\pi}{\beta-\alpha}$. If $f(z)$ has 
no Hayman $T$ direction in the angular domain $\Omega(\alpha, \beta)$, then the following real function satisfies $\lim _{r \rightarrow \infty} \Lambda(r)=0$, where $\Lambda(r)$ is defined as follows

$\Lambda(r)^{2}=\max \left\{\frac{\mathcal{T}\left(r_{n}, \Omega_{\varepsilon}, f\right)}{T\left(r_{n}, f\right)}, \frac{r_{n}^{\omega}}{T\left(r_{n}, f\right)} \int_{1}^{r_{n}} \frac{\mathcal{T}\left(t, \Omega_{\varepsilon}, f\right)}{t^{\omega+1}} d t, \frac{r_{n}^{\omega}\left[\log r_{n}+\log T\left(r_{n}, f\right)\right]}{T\left(r_{n}, f\right)}\right\}$

for $r_{n} \leq r<r_{n+1}$.

Proof. We should treat two cases.

Case (I). If there is no Hayman $T$ direction on $\Omega$, then from Lemma 2.5, we have

$$
\mathcal{T}\left(r, \Omega_{\varepsilon}, f\right)=o(T(2 r, f))+O\left(\log ^{3} r\right) \text { as } r \rightarrow \infty .
$$

Combining Lemma 2.1 and $\sigma>\omega$, we have

$$
\begin{aligned}
\int_{1}^{r_{n}} \frac{\mathcal{T}\left(t, \Omega_{\varepsilon}, f\right)}{t^{\omega+1}} d t & =o\left(\int_{1}^{r_{n}} \frac{T(2 t, f)}{t^{\omega+1}} d t\right)+\int_{1}^{r_{n}} \frac{O\left(\log ^{3} t\right)}{t^{\omega+1}} d t \\
& \leq o\left(\int_{1}^{r_{n}} \frac{T\left(r_{n}, f\right)}{t^{\omega+1}}\left(\frac{2 t}{r_{n}}\right)^{\sigma} d t\right)+O\left(\log ^{3} r_{n}\right) \\
& =o\left(\frac{T\left(r_{n}, f\right)}{r_{n}^{\omega}}\right)+O\left(\log ^{3} r_{n}\right) .
\end{aligned}
$$

Then

Case (II). If

$$
\frac{r_{n}^{\omega}}{T\left(r_{n}, f\right)} \int_{1}^{r_{n}} \frac{\mathcal{T}\left(t, \Omega_{\varepsilon}\right)}{t^{\omega+1}} d t \rightarrow 0 \quad \text { as } n \rightarrow \infty .
$$

then by (2.2), we have

$$
\limsup _{n \rightarrow \infty} \frac{\mathcal{T}\left(r_{n}, \Omega_{\varepsilon}, f\right)}{T\left(r_{n}, f\right)}>0
$$

$$
\limsup _{n \rightarrow \infty} \frac{N\left(2 r_{n}, \Omega, f=a\right)+N\left(2 r_{n}, \Omega, f^{(k)}=b\right)}{T\left(r_{n}, f\right)}>0 .
$$

Since $\left\{r_{n}\right\}$ is a sequence of Pólya peaks of order $\sigma$, then we have

$$
T\left(2 r_{n}, f\right) \leq 2^{\sigma} T\left(r_{n}, f\right) .
$$

Then $\Omega$ must contain a Hayman $T$ direction of $f(z)$. This is a contradiction to the hypothesis.

From Case (I) and Case (II) and notice that $r_{n}^{\omega}\left[\log r_{n}+\log T\left(r_{n}, f\right)\right] / T\left(r_{n}, f\right)$ $\rightarrow 0(n \rightarrow \infty)$, we have proved that $\limsup _{r \rightarrow \infty} \Lambda(r)=0$.

The following result was firstly established by Zheng [10, Theorem 2.4.7], it is crucial for our study.

Lemma 2.7. Let $f(z)$ be a function meromorphic on $\Omega=\Omega(\alpha, \beta)$. Then

$$
S_{\alpha, \beta}(r, f) \leq 2 \omega^{2} \frac{\mathcal{T}(r, \Omega, f)}{r^{\omega}}+\omega^{3} \int_{1}^{r} \frac{\mathcal{T}(t, \Omega, f)}{t^{\omega+1}} d t+O(1), \quad \omega=\frac{\pi}{\beta-\alpha} .
$$

We also have to use the following lemma, which is due to Hayman and Miles $[4]$. 
Lemma 2.8. Let $f(z)$ be meromorphic in the complex plane. Then for a given $K>1$, there exists a set $M(K)$ with $\overline{\log \operatorname{dens}} M(K) \leq \delta(K), \delta(K)=$ $\min \left\{\left(2 e^{K-1}-1\right)^{-1},(1+e(K-1) \exp (e(1-K)))\right\}$, such that

$$
\limsup _{r \rightarrow+\infty, r \notin M(K)} \frac{T(r, f)}{T\left(r, f^{(p)}\right)} \leq 3 e K .
$$

\section{Proof of Theorem 1.2}

Case (I). $\lambda(f)>\mu$. Then we choose $\sigma$ such that $\lambda\left(f^{(p)}\right)=\lambda(f)>\sigma \geq \mu=$ $\mu\left(f^{(p)}\right), \sigma>\omega$. From the inequality (1.5), we can take a real number $\varepsilon>0$ such that

$$
\sum_{j=1}^{q}\left(\alpha_{j+1}-\beta_{j}+4 \varepsilon\right)+\varepsilon<\sum_{j=1}^{l} \frac{4}{\sigma+2 \varepsilon} \arcsin \sqrt{\frac{\delta\left(a_{j}, f^{(p)}\right)}{2}}
$$

and

$$
\lambda\left(f^{(p)}\right)>\sigma+2 \varepsilon>\mu .
$$

Then there exists a sequence of Pólya peaks $\left\{r_{n}\right\}$ of order $\sigma+2 \varepsilon$ of $f^{(p)}$ such that $\left\{r_{n}\right\}$ is not in the set of Lemma 2.4 and Lemma 2.8.

We define $q$ real functions $\Lambda_{j}(r)(j=1,2, \ldots, q)$ as follows:

$$
\begin{aligned}
& \Lambda_{j}(r)^{2} \\
= & \max \left\{\frac{\mathcal{T}\left(r_{n}, \Omega\left(\alpha_{j}+\varepsilon, \beta_{j}-\varepsilon\right), f\right)}{T\left(r_{n}, f\right)}, \frac{r_{n}^{\omega_{j}}}{T\left(r_{n}, f\right)} \int_{1}^{r_{n}} \frac{\mathcal{T}\left(t, \Omega\left(\alpha_{j}+\varepsilon, \beta_{j}-\varepsilon\right), f\right)}{t^{\omega_{j}+1}} d t, \frac{r_{n}^{\omega_{j}}\left[\log r_{n}+\log T\left(r_{n}, f\right)\right]}{T\left(r_{n}, f\right)}\right\}
\end{aligned}
$$

for $r_{n} \leq r<r_{n+1}, \omega_{j}=\frac{\pi}{\beta_{j}-\alpha_{j}}$. By using Lemma 2.5, we have $\Lambda_{j}(r) \rightarrow 0$, as $r \rightarrow \infty$, if $f(z)$ has no Hayman $T$ directions on $X$. Set $\Lambda(r)=\max _{1 \leq j \leq q}\left\{\Lambda_{j}(r)\right\}$, then $\lim _{r \rightarrow \infty} \Lambda(r)=0$. Therefore for large enough $n$, by Lemma 2.2 we have

$$
\sum_{j=1}^{l} \operatorname{meas} D_{\Lambda}\left(r_{n}, a_{j}\right)>\min \left\{2 \pi, \frac{4}{\sigma+2 \varepsilon} \sum_{j=1}^{l} \arcsin \sqrt{\frac{\delta\left(a_{j}, f^{(p)}\right)}{2}}\right\}-\varepsilon
$$

Note that $\sigma+2 \varepsilon>1 / 2$. We can suppose for any $n(3.2)$ holds. Set

$$
K_{n}=\operatorname{meas}\left(\left(\bigcup_{j=1}^{l} D_{\Lambda}\left(r_{n}, a_{j}\right)\right) \bigcap\left(\bigcup_{j=1}^{q}\left(\alpha_{j}+2 \varepsilon, \beta_{j}-2 \varepsilon\right)\right)\right) .
$$

Combining (3.1) with (3.2), we obtain

$$
\begin{aligned}
K_{n} & \geq \sum_{j=1}^{l} \operatorname{meas}\left(D_{\Lambda}\left(r_{n}, a_{j}\right)\right)-\operatorname{meas}\left([-\pi, \pi) \backslash \bigcup_{j=1}^{q}\left(\alpha_{j}+2 \varepsilon, \beta_{j}-2 \varepsilon\right)\right) \\
& =\sum_{j=1}^{l} \operatorname{meas}\left(D_{\Lambda}\left(r_{n}, a_{j}\right)\right)-\operatorname{meas}\left(\bigcup_{j=1}^{q}\left(\beta_{j}-2 \varepsilon, \alpha_{j+1}+2 \varepsilon\right)\right)
\end{aligned}
$$




$$
=\sum_{j=1}^{l} \operatorname{meas}\left(D_{\Lambda}\left(r_{n}, a_{j}\right)\right)-\sum_{j=1}^{q}\left(\alpha_{j+1}-\beta_{j}+4 \varepsilon\right)>\varepsilon>0 .
$$

There exists a $j_{0}$ such that for infinitely many $n$

$$
\operatorname{meas}\left(\bigcup_{j=1}^{l} D_{\Lambda}\left(r_{n}, a_{j}\right) \bigcap\left(\alpha_{j_{0}}+2 \varepsilon, \beta_{j_{0}}-2 \varepsilon\right)\right)>\frac{K_{n}}{q}>\frac{\varepsilon}{q} .
$$

We can assume that the above holds for all the $n$.

Set $E_{n j}=D\left(r_{n}, a_{j}\right) \bigcap\left(\alpha_{j_{0}}+2 \varepsilon, \beta_{j_{0}}-2 \varepsilon\right)$. Thus we have

$$
\begin{aligned}
& \sum_{j=1}^{l} \int_{\alpha_{j_{0}}+2 \varepsilon}^{\beta_{j_{0}}-2 \varepsilon} \log ^{+} \frac{1}{\left|f^{(p)}\left(r_{n} e^{i \theta}\right)-a_{j}\right|} d \theta \\
\geq & \sum_{j=1}^{l} \int_{E_{n j}} \log ^{+} \frac{1}{\left|f^{(p)}\left(r_{n} e^{i \theta}\right)-a_{j}\right|} d \theta \\
\geq & \sum_{j=1}^{l} \operatorname{meas}\left(E_{n j}\right) \Lambda\left(r_{n}\right) T\left(r_{n}, f^{(p)}\right) \\
> & \frac{\varepsilon}{q} \Lambda\left(r_{n}\right) T\left(r_{n}, f^{(p)}\right) \\
> & \frac{\varepsilon}{3 e q K} \Lambda\left(r_{n}\right) T\left(r_{n}, f\right) .
\end{aligned}
$$

The last inequality uses Lemma 2.8.

On the other hand, we have

$$
\begin{aligned}
& \sum_{j=1}^{l} \int_{\alpha_{j_{0}}+2 \varepsilon}^{\beta_{j_{0}}-2 \varepsilon} \log ^{+} \frac{1}{\left|f(p)\left(r_{n} e^{i \theta}\right)-a_{j}\right|} d \theta \\
\leq & \sum_{j=1}^{l} \frac{\pi}{2 \omega_{j_{0}} \sin \left(\varepsilon \omega_{j_{0}}\right)} r_{n}^{\omega_{j_{0}}} B_{\alpha_{j_{0}}+\varepsilon, \beta_{j_{0}}-\varepsilon}\left(r_{n}, \frac{1}{f(p)-a_{j}}\right) \\
< & \sum_{j=1}^{l} \frac{\pi}{2 \omega_{j_{0}} \sin \left(\varepsilon \omega_{j_{0}}\right)} r_{n}^{\omega_{j_{0}}} S_{\alpha_{j_{0}}+\varepsilon, \beta_{j_{0}}-\varepsilon}\left(r_{n}, \frac{1}{f(p)-a_{j}}\right) \\
= & \frac{l \pi}{2 \omega_{j_{0}} \sin \left(\varepsilon \omega_{j_{0}}\right)} r_{n}^{\omega_{j_{0}}} S_{\alpha_{j_{0}}+\varepsilon, \beta_{j_{0}}-\varepsilon}\left(r_{n}, f^{(p)}\right)+O\left(r_{n}^{\omega_{j_{0}}}\right) \\
\leq & \frac{l \pi}{2 \omega_{j_{0}} \sin \left(\varepsilon \omega_{j_{0}}\right)} r_{n}^{\omega_{j_{0}}}\left[(p+1) S_{\alpha_{j_{0}}+\varepsilon, \beta_{j_{0}}-\varepsilon}\left(r_{n}, f\right)+\log r_{n}+\log T\left(r_{n}, f\right)\right] \\
& +O\left(r_{n}^{\omega_{j_{0}}}\right) \\
\leq & \frac{l \pi}{2 \omega_{j_{0}} \sin \left(\varepsilon \omega_{j_{0}}\right)}(p+1)\left[2 \omega_{j_{0}}^{2} \mathcal{T}\left(r_{n}, \Omega\left(\alpha_{j_{0}}+\varepsilon, \beta_{j_{0}}-\varepsilon\right), f\right)\right.
\end{aligned}
$$




$$
\begin{aligned}
& \left.+\omega_{j_{0}}^{3} r_{n}^{\omega_{j_{0}}} \int_{1}^{r_{n}} \frac{\mathcal{T}\left(t, \Omega\left(\alpha_{j_{0}}+\varepsilon, \beta_{j_{0}}-\varepsilon\right), f\right)}{t^{\omega_{j_{0}}}+1} d t\right] \\
& +\frac{l \pi}{2 \omega_{j_{0}} \sin \left(\varepsilon \omega_{j_{0}}\right)} r_{n}^{\omega_{j_{0}}}\left[\log r_{n}+\log T\left(r_{n}, f\right)\right]+O\left(r_{n}^{\omega_{j_{0}}}\right) \\
\leq & \frac{l \pi}{2 \omega_{j_{0}} \sin \left(\varepsilon \omega_{j_{0}}\right)}(p+1)\left[2 \omega_{j_{0}}^{2} \Lambda\left(r_{n}\right)^{2} T\left(r_{n}, f\right)+\omega_{j_{0}}^{3} \Lambda\left(r_{n}\right)^{2} T\left(r_{n}, f\right)\right] \\
& +\frac{l \pi}{2 \omega_{j_{0}} \sin \left(\varepsilon \omega_{j_{0}}\right)} r_{n}^{\omega_{j_{0}}}\left[\log r_{n}+\log T\left(r_{n}, f\right)\right]+O\left(r_{n}^{\omega_{j_{0}}}\right), \omega_{j_{0}}=\frac{\pi}{\beta_{j_{0}}-\alpha_{j_{0}}-2 \varepsilon}
\end{aligned}
$$

(3.3) and (3.4) imply that

$$
\Lambda\left(r_{n}\right) \leq O\left(\Lambda\left(r_{n}\right)^{2}\right)
$$

A contradiction is derived because $\Lambda\left(r_{n}\right) \rightarrow 0$ as $n \rightarrow \infty$.

Case (II). $\lambda(f)=\mu$. By the same argument as in Case (I) with all the $\sigma+2 \varepsilon$ replaced by $\sigma=\mu$, we can derive the same contradiction.

Acknowledgements. Nan $\mathrm{Wu}$ is supported in part by the grants of NSF of China (Nos.11231009, 11326086, 11371363). Zuxing Xuan is supported in part by Beijing Natural Science Foundation (No.1132013), The Importation and Development of High-Caliber Talents Project of Beijing Municipal Institutions(CIT and TCD201504041) and NSF of China (No.91420202).

\section{References}

[1] A. A. Goldberg and I. V. Ostrovskii, The Distribution of Values of Meromorphic Functions, Izdat. Nauk. Moscow, 1970.

[2] H. Guo, J. H. Zheng, and T. W. Ng, On a new singular direction of meromorphic functions, Bull. Austral. Math. Soc. 69 (2004), no. 2, 277-287.

[3] W. K. Hayman, Meromorphic Functions, Clarendon, Press, Oxford, 1964.

[4] W. K. Hayman and Miles, On the growth of a meromorphic function and its derivatives, Complex Variables Theory Appl. 12 (1989), no. 1-4, 245-260.

[5] M. Tsuji, Potential Theory in Modern Function Theory, Maruzen Co., Ltd Tokyo, 1959.

[6] S. Wang, On some properties of Fatou and Julia sets of meromorphic functions, Dissertation submitted to Tsinghua University in partial fulfillment of the requirements for the degree of Doctor of Natural Science, 2002.

[7] L. Yang, Value Distribution and New Research, Springer-Verlag, Berlin, 1993.

8] - Borel directions of meromorphic functions in an angular domain, Sci. Sinica (1979), 149-164.

[9] Q. D. Zhang, T directions and Borel directions of meromorphic functions of finite and positive order, Acta Math. Sinica 50 (2007), no. 2, 413-420.

[10] J. H. Zheng, Value Distribution of Meromorphic Functions, Tsinghua University Press, Beijing, 2010.

[11] - On transcendental meromorphic functions with radially distributed values, Sci. China Ser. A. Math. 47 (2004), no. 3, 401-416.

[12] J. H. Zheng and N. Wu, Hayman T directions of meromorphic functions, Taiwanese J. Math. 14 (2010), no. 6, 2219-2228. 
NAN WU

Department of Mathematics

SCHOOL OF SCIENCE

China University of Mining and Technology(Beijing)

BeiJing, 100083, P. R. China

E-mail address: wunan2007@163.com

ZU-XING XUAN

Beijing Key Laboratory of Information Service Engineering Department of General Education

Beijing Union University, No.97 Bei Si Huan Dong Road

Chaoyang District, Beijing, 100101, P. R. China

E-mail address: xuanzuxing@ss.buaa.edu.cn 